\title{
Effective models of WIMP Direct Detection in DAMA/LIBRA-phase2
}

\author{
Sunghyun Kang, Stefano Scopel, Gaurav Tomar, Jong-Hyun Yoon* \\ Department of Physics, Sogang University, Seoul, Korea, 121-742 \\ E-mail: francis735enaver.com, scopel@sogang.ac.kr, \\ tomaresogang.ac.kr, jyoonesogang.ac.kr
}

\begin{abstract}
Since the DAMA/LIBRA collaboration released updated results from their search for the annual modulation signal expected from Dark Matter (DM) scattering in their NaI detectors, we have fitted the updated DAMA result for the modulation amplitudes for a Weakly Interacting Massive Particle (WIMP) signal, parameterizing the interaction with nuclei in terms of the most general effective Lagrangian for a WIMP particle spin 1/2, systematically assuming dominance of one of the 14 possible interaction terms and a standard Maxwellian for the WIMP velocity distribution. We find that most of the couplings of the non-relativistic effective Hamiltonian can provide a better fit compared to the standard Spin Independent interaction case, and with a reduced finetuning of the three parameters (WIMP mass, WIMP-nucleon effective cross-section and ratio between the WIMP-neutron and the WIMP-proton couplings). In addition, effective models for which the cross section depends explicitly on the WIMP incoming velocity can provide a better fit of the DAMA data at large values of $m_{\chi}$ compared to the standard velocity-independent crosssection due to a different phase of the modulation amplitudes. All the best fit solutions are in tension with exclusion plots of both XENON1T and PICO60.
\end{abstract}

ICHEP 2018, XXXIX International Conference on High Energy Physics

4-11 July 2018

COEX, Seoul, Korea

${ }^{*}$ Speaker. 


\section{Introduction}

In this work we will consider the most general WIMP (Weakly Interacting Massive Particle) -nucleus effective Lagrangian for a WIMP particle of spin 1/2 scattering elastically off nuclei, systematically assuming the dominance of one of the 14 possible interaction terms of the most general non-relativistic Hamiltonian invariant by Galilean transformations, fitting the new DAMA data to the three parameters $m_{\chi}$ (WIMP mass), $\sigma_{p}$ (WIMP-nucleon effective cross-section) and $c^{n} / c^{p}$ (WIMP-neutron/proton coupling ratio).

\section{WIMP rates in non-relativistic effective models}

Making use of the non-relativistic EFT approach one can write the most general Hamiltonian density describing the WIMP-nucleus interaction as:

$$
\mathscr{H}(\mathbf{r})=\sum_{\tau=0,1} \sum_{j=1}^{15} c_{j}^{\tau} \mathscr{O}_{j}(\mathbf{r}) t^{\tau}
$$

where $\mathscr{O}_{j}$ can be found in [2]. Assuming that the nuclear interaction is the sum of the interactions of the WIMPs with the individual nucleons in the nucleus the WIMP scattering amplitude on the target nucleus $T$ can be written in the compact form:

$$
\frac{1}{2 j_{\chi}+1} \frac{1}{2 j_{T}+1}|\mathscr{M}|^{2}=\frac{4 \pi}{2 j_{T}+1} \sum_{\tau=0,1} \sum_{\tau^{\prime}=0,1} \sum_{k} R_{k}^{\tau \tau^{\prime}}\left[c_{j}^{\tau},\left(v_{T}^{\perp}\right)^{2}, \frac{q^{2}}{m_{N}^{2}}\right] W_{T k}^{\tau \tau^{\prime}}(y) .
$$

In the above expression $j_{\chi}$ and $j_{T}$ are the WIMP and the target nucleus spins, respectively, $q=|\vec{q}|$ while the $R_{k}^{\tau \tau^{\prime}}$,s are WIMP response functions which depend on the couplings $c_{j}^{\tau}$ as well as the transferred momentum $\vec{q}$ and $\left(v_{T}^{\perp}\right)^{2}$. In equation (2.2) the $W_{T k}^{\tau \tau^{\prime}}(y)$ 's are nuclear response functions and the index $k$ represents different effective nuclear operators, which can be at most eight: $k=M$, $\Phi^{\prime \prime}, \Phi^{\prime \prime} M, \tilde{\Phi}^{\prime}, \Sigma^{\prime \prime}, \Sigma^{\prime}, \Delta, \Delta \Sigma^{\prime}$. The $W_{T k}^{\tau \tau^{\prime}}(y)$ 's are function of $y \equiv(q b / 2)^{2}$, where $b$ is the size of the nucleus. For the target nuclei $T$ used in most direct detection experiments the functions $W_{T k}^{\tau \tau^{\prime}}(y)$ have been calculated using nuclear shell models [2]. We systematically consider the possibility that one of the couplings $c_{j}$ dominates in the effective Hamiltonian of Eq. (2.1). In this case it is possible to factorize a term $\left|c_{j}^{p}\right|^{2}$ from the squared amplitude of Eq.(2.2) and express it in terms of the effective WIMP-proton cross section:

$$
\sigma_{p}=\left(c_{j}^{p}\right)^{2} \frac{\mu_{\chi \mathscr{N}}^{2}}{\pi},
$$

(with $\mu_{\chi \mathscr{N}}$ the WIMP-nucleon reduced mass) and the ratio $r \equiv c_{j}^{n} / c_{j}^{p}$.

\section{Analysis}

We perform a $\chi^{2}$ analysis constructing the quantity: 


$$
\chi^{2}\left(m_{\chi}, \sigma_{p}, r\right)=\sum_{k=1}^{15} \frac{\left[S_{m, k}-S_{m, k}^{e x p}\left(m_{\chi}, \sigma_{p}, r\right)\right]^{2}}{\sigma_{k}^{2}}
$$

and minimize it as a function of $\left(m_{\chi}, \sigma_{p}, r\right)$. In the equation above $S_{m, k}$ and $\sigma_{k}$ represent the modulation amplitudes and errors measured by DAMA, while $S_{m, k}^{\exp }$ denotes the expected modulation rate with respect to $\left(m_{\chi}, \sigma_{p}, r\right)$. In Fig. 1 we show the result of such minimization at fixed WIMP mass $m_{\chi}$. The details of such minima in the particular example of $c_{1}$ and $c_{5}$ are provided in Table 1 . The best fit parameters for $c_{5}$ appear to be less tuned compared to the SI case. This can be seen in Fig. 2, where we provide the contour plots of the $\chi^{2}$ in the $m_{\chi}-r$ plane for $c_{1}$ and $c_{5}$. In particular, the regions within 2 and $3 \sigma$ for $c_{1}$ appear strongly tuned to the value $r=-0.76$, corresponding to a cancellation in the WIMP-iodine cross section, whereas for $c_{5}$ the corresponding contour encompasses a much wider volume of the parameter space.

\begin{tabular}{|c|c|c|c|c|}
\hline $\mathbf{c}_{\mathbf{j}}$ & $\mathbf{m}_{\chi, \min }(\mathbf{G e V})$ & $\mathbf{r}_{\chi, \mathbf{m i n}}$ & $\sigma\left(\mathbf{c m}^{\mathbf{2}}\right)$ & $\chi_{\min }^{\mathbf{2}}$ \\
\hline$c_{1}$ & 11.17 & -0.76 & $2.67 \mathrm{e}-38$ & 11.38 \\
$c_{5}$ & 8.34 & -0.61 & $1.62 \mathrm{e}-29$ & 10.83 \\
\hline
\end{tabular}

Table 1: Absolute minima of the $\chi^{2}$ (see Eq.(3.1)) for the couplings $c_{1}$ and $c_{5}$ of the effective Hamiltonian (2.1). See [3] for a full set of contents in the table.
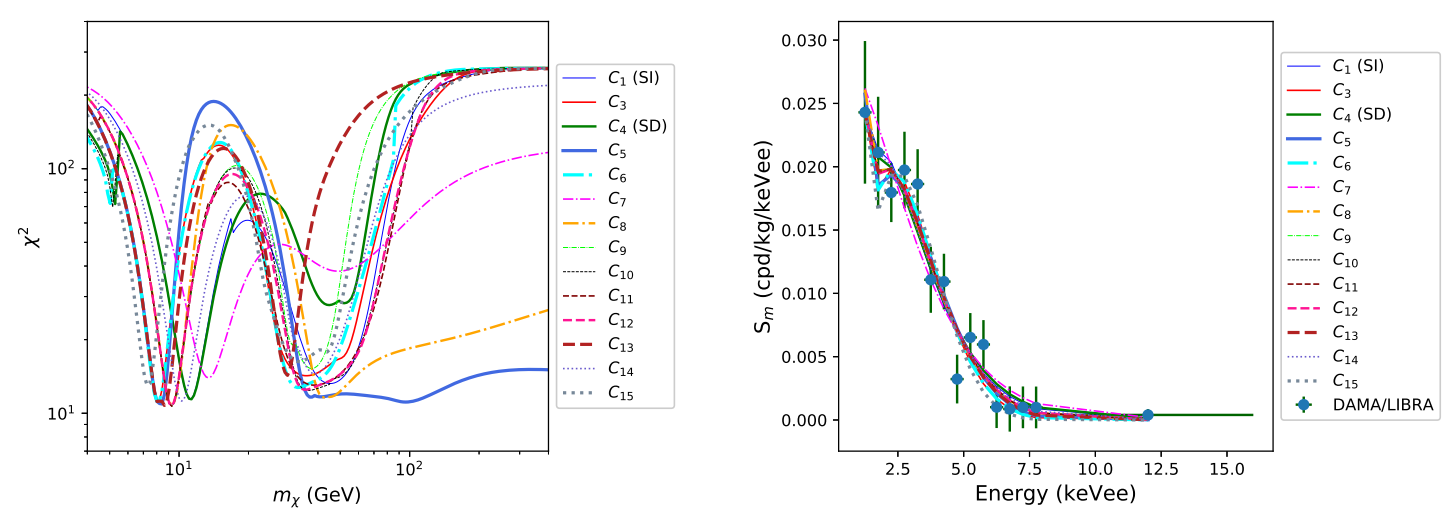

Figure 1: Minimum of the $\chi^{2}$ of Eq.(3.1) at fixed WIMP mass $m_{\chi}$ as a function of $m_{\chi}$ for different WIMPnucleus interactions (Left). DAMA modulation amplitudes as a function of the measured ionization energy $E_{e e}$ for the absolute minima of each effective model (Right). The points with error bars correspond to the combined data of DAMA/NaI, DAMA/LIBRA-phase1 and DAMA/LIBRA-phase2 [1]

In the case of $c_{5}$ the velocity-independent term of the cross section depends on the $\Delta$ response function, which is proportional to the nucleon angular momentum content of the nucleus, favoring elements which have an unpaired nucleon in a non $s$-shell orbital. Both iodine and sodium have this feature, implying in this case no large hierarchy between the cross sections off the two nuclei, hence less fine-tuning. Namely, numerically the isoscalar response function at vanishing momentum transfer $W_{T \Delta}^{00}(q \rightarrow 0)$ for sodium is a factor $\simeq 0.25$ smaller compared to that for iodine. As can be 
seen from Fig. 1 for most models the $\chi^{2}$ shows a steep rise at large $m_{\chi}$. In these cases, the predicted modulation amplitude is given by the cosine transform of the rate, which is a function of the $v_{\min }$ parameter only, and turns out to be negative for $v_{\min } \lesssim 200 \mathrm{~km} / \mathrm{sec}$. This implies a bad fit to the data and a large $\chi^{2}$. The situation is different when the cross section shows a non-negligible dependence on $v^{\perp}$, (i.e. for models such as $c_{5}$ ). In this case the integral of the differential rate is dominated by large values of $v>200 \mathrm{~km} / \mathrm{sec}$ irrespective of $v_{\min }$ and positive modulation amplitudes can be obtained in the energy range of the DAMA signal also at large values of $m_{\chi}$, implying in such regime a milder increase of the $\chi^{2}$ (or even an acceptable fit in the specific case of $c_{5}$ ).
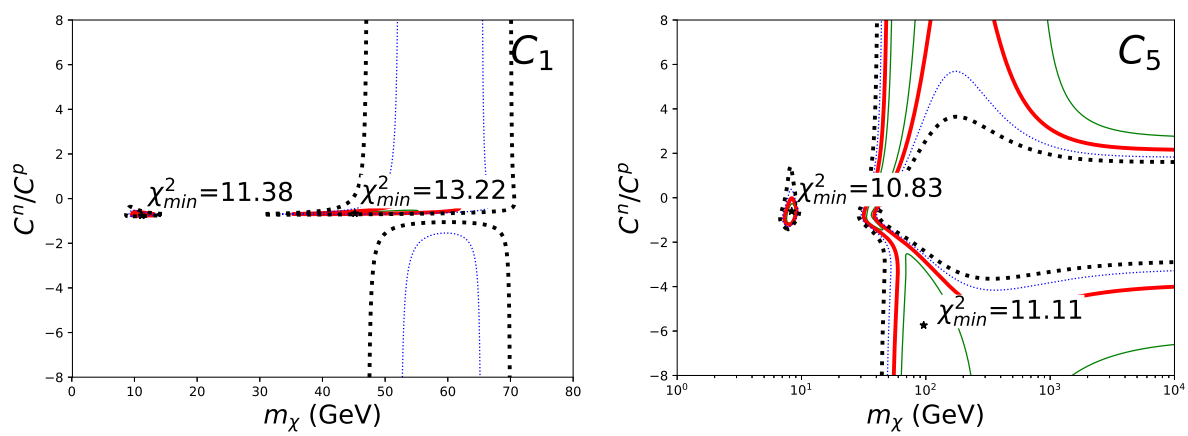

Figure 2: Contour plots of the $\chi^{2}$ of Eq.(3.1) minimized with respect to $\sigma_{p}$ in the $m_{\chi^{-}} r$ plane for interactions $c_{1}$ and $c_{5}$. The thin (green) solid lines, the thick (red) solid lines, the thin (blue) dotted lines, and the thick (black) dotted lines correspond to 2, 3, 4, and $5 \sigma$ regions respectively. See [3] for complete analysis.

\section{Conclusions}

The DAMA collaboration has released first results from the upgraded DAMA/LIBRA-phase2 experiment [1]. Assuming the dominance of one of the 14 possible interaction terms of the Hamiltonian of Eq.(2.1), we have fitted the experimental amplitudes to the three parameters $m_{\chi}$ (WIMP mass), $\sigma_{p}$ (WIMP-nucleon effective cross-section) and $c^{n} / c^{p}$ (neutron over proton coupling) with the WIMP velocity distribution a standard Maxwellian. In Section 3, we have discussed the phenomenological aspects of two particular effective operators, $c_{1}$ and $c_{5}$, in light of the results from DAMA/LIBRA-phase2. In [3], we have studied all the other effective couplings of the Hamiltonian of Eq. (2.1) and found that all the best fit solutions are in tension with exclusion plots of both XENON1T and PICO60.

\section{References}

[1] R. Bernabei et al., First model independent results from DAMA/LIBRA-phase2, arXiv:1805.10486.

[2] A. L. Fitzpatrick, W. Haxton, E. Katz, N. Lubbers, and Y. Xu, The Effective Field Theory of Dark Matter Direct Detection, JCAP 1302 (2013) 004, [arXiv: 1203.3542 ].

[3] S. Kang, S. Scopel, G. Tomar, and J.-H. Yoon, DAMA/LIBRA-phase2 in WIMP effective models, JCAP 1807 (2018), no. 07 016, [arXiv: 1804.07528 ]. 